\title{
Modal Strain Based Method for Dynamic Design of Plate-Like Structures
}

\author{
Yadong Zhou, ${ }^{1,2}$ Shaoqing Wu, ${ }^{1,3}$ Natasa Trisovic, ${ }^{4}$ Qingguo Fei, ${ }^{1,3}$ and Zhiyong Tan ${ }^{5}$ \\ ${ }^{1}$ Department of Engineering Mechanics, Southeast University, Nanjing 210096, China \\ ${ }^{2}$ Department of Civil and Environmental Engineering and Department of Mechanical Engineering, \\ Northwestern University, Evanston, IL 60208, USA \\ ${ }^{3}$ Jiangsu Key Laboratory of Engineering Mechanics, Nanjing 210096, China \\ ${ }^{4}$ Department of Mechanics, University of Belgrade, 11120 Belgrade, Serbia \\ ${ }^{5}$ State Key Laboratory of Space Physics, Beijing 100076, China \\ Correspondence should be addressed to Qingguo Fei; qgfei@seu.edu.cn
}

Received 4 July 2016; Revised 22 September 2016; Accepted 17 October 2016

Academic Editor: Carlo Rainieri

Copyright (C) 2016 Yadong Zhou et al. This is an open access article distributed under the Creative Commons Attribution License, which permits unrestricted use, distribution, and reproduction in any medium, provided the original work is properly cited.

\begin{abstract}
Design optimization of dynamic properties, for example, modal frequencies, can be of much importance when structures are exposed to the shock and/or vibration environments. A modal strain based method is proposed for fast design of natural frequencies of plate-like structures. The basic theory of modal strains of thin plates is reviewed. The capability of determining the highly sensitive elements by means of modal strain analysis is theoretically demonstrated. Finite element models were constructed in numerical simulations. Firstly, the application of the proposed method is conducted on a central-massed flat plate which was topologically optimized by the Reference. The results of modal strain analysis at the first mode have good agreement with the results from the topology optimization. Furthermore, some features of the strain mode shapes (SMSs) of the flat plate are investigated. Finally, the SMSs are applied to the optimization of a stiffened plate. Attention is focused on the distributions of the SMSs of the stiffeners, which also shows good agreement with the results from the topology optimization in the previous study. Several higher orders of SMSs are extracted, which can visualize the most sensitive elements to the corresponding modal frequency. In summary, both the theory and simulations validate the correctness and convenience of applying SMSs to dynamic design of plate-like structures.
\end{abstract}

\section{Introduction}

Plate-like structures are widely used in the areas of aeronautics, automobile engineering and ship structures, due to their light weight and high structural efficiency, and so forth. However, the thin plate-like structures can be characterized by low flexural rigidity. The dynamic behaviours of these structures are important properties, because the natural frequencies and mode shapes of the thin plates usually need to satisfy the requirements of structural integrity, durability, and sound radiation when subjected to the shock and vibration loadings, which matters in different branches of engineering: automobile [1, 2], naval [3], aeronautics and astronautics [4], and so forth.
The goal of the dynamic design is to avoid resonance of the structure in a given interval for external excitation frequencies. This can be achieved by, for example, maximizing the fundamental frequency, increasing an modal frequency of a higher order, or the gap between two consecutive modal frequencies of given orders [5]. The mechanical performances of the load-carrying plate-like structures, including the stiffness, structural strength, dynamic characteristics, and vibration fatigue, are highly dependent on the thickness distributions and stiffener layout patterns; and hence various computational approaches have been developed to predict the optimal thickness distributions [6], geometric parameters [7], or stiffener layout patterns [8-10] for plate-like structures. 
Design of the dynamic behaviours can be formulated as a case of reinforcement optimization, where parts of the structure are fixed to be solid for purpose of avoiding a trivial solution. As a specific example of reinforcement optimization, dynamic design of the stiffened thin plates can be formulated as the optimal design of the rib-reinforcement of plates. Eigenvalue sensitivity analysis has been conducted as a powerful tool for structural dynamic modification [11] and design [12] and so forth. The eigenvalue sensitivity with respect to the locations of stiffeners can also be useful in the optimization of stiffened plates [13]. More generally, maximization of eigenvalues using topology optimization is considered as an effective and popular approach $[14,15]$. The main idea of the topology optimization is the removal of ineffective (e.g., comparatively small stressed) elements from the design domain. In order to find accurate solution of topology optimization, the designer must increase the number of iteration and the number of elements. When the numbers of iteration and elements are increased, solution time also increases extremely. By transforming the design problem of stiffener layout patterns into a search for an optimal distribution of materials, a very large number of design variables are required in order to represent the shape in the most general way. Consequently, expensive computations are required. These can be somewhat time-consuming during the preliminary design process.

As a progress of modal analysis method, curvature mode shapes (CMSs) and/or strain mode shapes (SMSs) have been developed theoretically, numerically, and experimentally for several decades [16-21]. Yam et al. [17] revealed that the strain mode can be more sensitive to local changes of the structure than the displacement mode. By a comparison of strain and classic experimental modal analysis (EMA), Kranjc et al. [20] pointed out that strain EMA can offer advantages which are important for particular applications; for example, the direct identification of SMSs can be highly important in the vibration-fatigue and damage-identification models. Hong et al. [22] conducted a comprehensive comparison of macrostrain mode and displacement mode obtained from distributed macrostrain sensing and high-density point sensing (e.g., accelerometers) technologies. Because of the highly sensitive characteristic to local defects, modal curvature and/or modal strain based quantities have been widely utilized to identify the presence, locations, and severity of damage in dynamic structures by the comparisons of before and after damage [23-26]. For the assessment of structural status, modal macrostrain vectors, macrostrain frequency response functions, and modal curvatures were successfully applied in structural health monitoring [27, 28]. Furthermore, for dynamic stresses analysis, Pelayo et al. [29] proposed a modal-based method in which the SMSs play a key role. In random fatigue evaluation, prognosis of fatigue hotspots by using stress mode shapes was theoretically demonstrated, and hence a two-step evaluation procedure was proposed for computational efficiency [30]. From the aforementioned literature, it can be seen that curvature/strain and stress mode shapes have their own advantages over displacement mode shapes due to the more sensitivity to local deformations and changes.
Continuing in this line of investigation, the modal strain theory is adopted to investigate the dynamic design of platelike structures. Strain mode shapes will be demonstrated to efficiently locate the most sensitive areas and hence to facilitate the fast design of plate thickness and/or stiffener layout for desired modal frequencies. In Section 2, the basic theory of modal strains is reviewed at first; then the formulation of eigenvalue sensitivity is presented and the correlation between the eigenvalue sensitivity and the modal strain distribution is theoretically derived. In Section 3, several numerical simulations are performed to investigate the basic law of the modal strain and its applicability in dynamic design of plates. Comparisons between the results of modal strain analysis and those of topology optimization are conducted. In Section 4, the conclusion is reached.

\section{Theoretical Background}

2.1. Modal Strains of Thin Plates. Considering the formulation for vibrational natural frequency which can be described by the following eigenvalue problem [31],

$$
\mathbf{K}(\mathbf{b}) \phi=\lambda \mathbf{M}(\mathbf{b}) \phi,
$$

where $\lambda$ and $\phi$ are the eigenvalue and eigenvector, respectively, and $\lambda=\omega^{2}$, where $\omega$ is the natural circular frequency, the global stiffness matrix $\mathbf{K}(\mathbf{b})$ is a function of the vector $\mathbf{b}$ including the design parameters. In addition, the global mass matrix $\mathbf{M}(\mathbf{b})$ is also a function of the design parameters. With plate thickness design, the structural mass matrix depends on the plate thickness. For a structure characterized by $n$ nodal degrees of freedom (DOFs), the set of solutions $\left[\phi_{1}, \phi_{2}, \ldots, \phi_{n}\right]$ is composed of the $n$ eigenvectors, that is, the displacement mode shapes (DMSs), and the number of mode shapes considered in the dynamic response analysis is usually much less than the total number of nodal DOFs for largescale structures.

In this study, the method is presented on a Kirchhoff plate. The surface strains and the transverse (out-of-plane) displacement $w(x, y)$ in a thin plate with small deflection are related by the following equation [32]:

$$
\boldsymbol{\varepsilon}=\left\{\begin{array}{c}
\varepsilon_{x} \\
\varepsilon_{y} \\
\gamma_{x y}
\end{array}\right\}=-z\left\{\begin{array}{c}
\frac{\partial^{2} w}{\partial x^{2}} \\
\frac{\partial^{2} w}{\partial y^{2}} \\
2 \frac{\partial^{2} w}{\partial x \partial y}
\end{array}\right\},
$$

where $z$ is the distance from the plate surface to the neutral surface of the plate, that is, the half thickness of the thin plate.

In light of the strain-displacement relationship, one can define the SMSs as the distribution of the in-plane strain components at the top or bottom surface of the plate according to the corresponding natural vibration mode. If a finite element formulation is adopted, the deflection at any arbitrary point of a plate element is given by [32]

$$
\mathbf{w}(x, y)=\mathbf{N}^{e}(x, y) \mathbf{w}^{e},
$$


where $\mathbf{N}^{e}(x, y)$ is the shape function matrix and $\mathbf{w}^{e}$ is a vector containing the displacement and rotations of the node $\left(w, \theta_{x}, \theta_{y}\right)$. Substituting (3) into (2), the strain vector at the element surface can be given by

$$
\boldsymbol{\varepsilon}(x, y)=-z \mathbf{B}^{e}(x, y) \mathbf{w}^{e},
$$

where $\mathbf{B}^{e}(x, y)$ is the strain matrix of the element containing the second derivatives of the shape functions. With the mode superposition method, the dynamic strains on the surface of the plate element can be expressed as

$$
\boldsymbol{\varepsilon}(x, y, t)=-z \mathbf{B}^{e}(x, y) \boldsymbol{\phi}^{e} \mathbf{q}(t),
$$

where the product $\mathbf{B}^{e}(x, y) \phi^{e}$ represents the curvature mode shape in the element and $\mathbf{q}(t)$ is the modal coordinate. Therefore, for an individual finite element, the strain mode shapes on the plate surface can be derived as [17]

$$
\phi_{\varepsilon}^{e}=-z \mathbf{B}^{e}(x, y) \phi^{e} .
$$

Equation (6) represents the modal strain formulation at a specific finite element. The formulation for the whole model of the plate structure holds the similar form. Considering a structure containing $m$ finite elements, (6) for the whole model of the structure becomes

$$
\left\{\begin{array}{c}
\phi_{\varepsilon, 1}^{e} \\
\phi_{\varepsilon, 2}^{e} \\
\vdots \\
\phi_{\varepsilon, m}^{e}
\end{array}\right\}=-z\left[\begin{array}{cccc}
\mathbf{B}_{1}^{e} & & & \\
& \mathbf{B}_{2}^{e} & & \\
& & \ddots & \\
& & & \mathbf{B}_{m}^{e}
\end{array}\right]\left\{\begin{array}{c}
\phi_{1}^{e} \\
\phi_{2}^{e} \\
\vdots \\
\phi_{m}^{e}
\end{array}\right\} .
$$

The relationship between the displacement vector $\phi^{e}$ in the local coordinate system and the displacement vector $\phi$ in the global coordinate system is given by

$$
\phi^{e}=\mathbf{T} \phi
$$

where $\mathbf{T}$ is the coordinate transform matrix. By transforming the displacement in the global coordinate system to the displacement in the local coordinate system, the strain mode shapes of each individual element in the whole structure can be represented in a compact form as

$$
\phi_{\varepsilon}^{e}=-z \mathbf{B}^{e} \mathbf{T} \phi .
$$

The vector $\phi$ denotes the structural displacement mode shapes in the global coordinate system. The product $\mathbf{B}^{e} \mathbf{T} \phi$ is the curvature mode shapes of the deflected neutral surface.

2.2. Modal Principal Strains. Since the strain vectors can include different components, for a specific order of mode, the modal strain can have different distributions depending on the strain component, for example, the strain mode shape in the $x$-and $y$-directions of a plate structure. It is noted that the three in-plane components of strains are of importance for plate-like structures, that is, the two normal strains and the one shear strain. In some cases, however, the derived quantities can be much more important. For instance, Pedersen [33] found that the optimal orientation of orthotropic materials depends on one nondimensional material parameter plus the ratio of the two principal strains. Bendsoe et al. [34] expressed the strain energy density in the frame of the principal strains for frame-independent consideration in structural compliance optimization.

Strain based parameters that are independent of the coordinate system, for example, strain invariants and principal strains are examples of such scalar parameters. The maximum principal strain can be used as an overall measure of the magnitude of the in-plane strains. If the largest normal strain is of most interest, the $r$ th modal principal (maximum and minimum) strains of the $i$ th element $\left(\phi_{\varepsilon}^{1,2}\right)_{i r}$ can be expressed as

$$
\begin{aligned}
\left(\phi_{\varepsilon}^{1,2}\right)_{i r}= & \frac{\left(\phi_{\varepsilon}^{x}\right)_{i r}+\left(\phi_{\varepsilon}^{y}\right)_{i r}}{2} \\
& \pm \sqrt{\left(\frac{\left(\phi_{\varepsilon}^{x}\right)_{i r}-\left(\phi_{\varepsilon}^{y}\right)_{i r}}{2}\right)^{2}+\left(\frac{\left(\phi_{\varepsilon}^{x y}\right)_{i r}}{2}\right)^{2}},
\end{aligned}
$$

where $\left(\phi_{\varepsilon}^{x}\right)_{i r},\left(\phi_{\varepsilon}^{y}\right)_{i r}$, and $\left(\phi_{\varepsilon}^{x y}\right)_{i r}$ are the $r$ th modal strains of the $i$ th element in the $x_{-}, y_{-}$, and $x y$-components, respectively. Therefore, once the $x^{-}, y$-, and $x y$-components of the modal strains are obtained, modal principal strains can be calculated using (10) for each order of the mode shape. The benefit of the modal principal strain is to provide a scalar quantity to characterize the magnitude of the modal strain field.

2.3. Eigenvalue Sensitivity. In order to update design variables toward the optimized solution, the optimality criteria need to determine how different values of the design variables influence the objective function under a given set of design constraints. Thus, sensitivity analysis is needed in topology optimization. The sensitivity of eigenvalue with respect to the vector of design variables $\mathbf{b}$ can be formulated as follows [31]:

$$
\frac{\partial \lambda}{\partial \mathbf{b}}=\frac{\partial}{\partial \mathbf{b}}\left[\boldsymbol{\phi}^{T} \mathbf{K}(\mathbf{b}) \boldsymbol{\phi}\right]-\lambda \frac{\partial}{\partial \mathbf{b}}\left[\boldsymbol{\phi}^{T} \mathbf{M}(\mathbf{b}) \boldsymbol{\phi}\right]
$$

where $\mathbf{K}(\mathbf{b})$ and $\mathbf{M}(\mathbf{b})$ are the global stiffness matrix and the global mass matrix, respectively. The superscript " $T$ " denotes the transpose of a matrix. If plate thickness is considered as a design variable and if piecewise-constant thickness is assumed, the design variables $\mathbf{b}$ become the plate thicknesses of the $m$ finite elements $\left(b_{1}, b_{2}, \ldots, b_{m}\right)$ in the whole model. The sensitivity with respect to one of the design thicknesses, $b_{i}$, can be expressed as [31]

$$
\frac{\partial \lambda}{\partial b_{i}}=\iint_{\Omega_{i}}\left[\frac{E b_{i}^{2}}{4\left(1-\mu^{2}\right)} \boldsymbol{\kappa}(w)^{T} \mathbf{C} \boldsymbol{\kappa}(w)-\lambda \rho w^{2}\right] d \Omega
$$

In the structural domain $\Omega, \rho$ denotes the mass density, $E$ is Young's modulus, and $\mu$ is Poisson's ratio. The curvature 


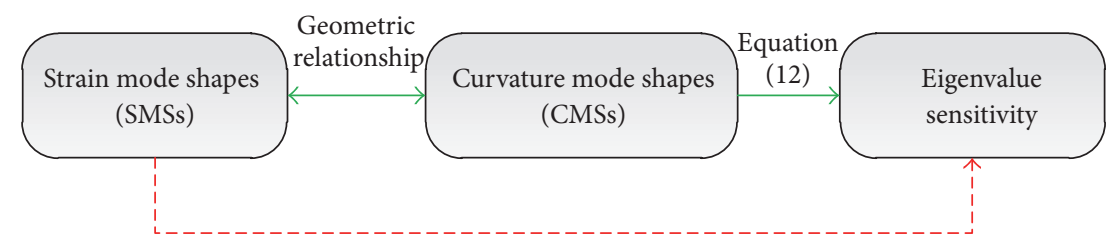

FIgURE 1: Relationships between the SMSs, CMSs, and eigenvalue sensitivity.

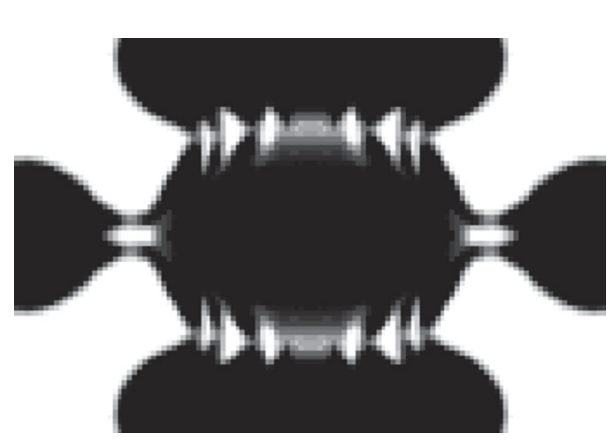

(a)

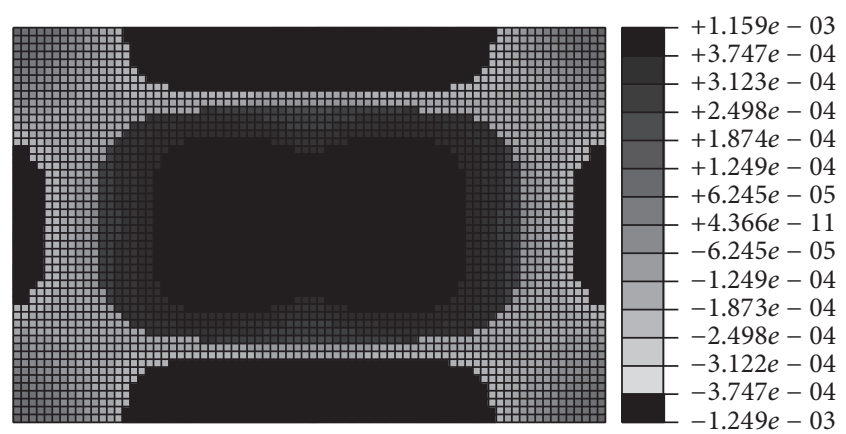

(b)

FIgURE 2: Topology optimization result and the modal principal strain of a clamped plate: (a) topology optimization result from [14]; (b) modal principal strain.

vector $\boldsymbol{\kappa}(w)$ and the matrix $\mathbf{C}$ for an isotropic material are, respectively, expressed as

$$
\begin{gathered}
\boldsymbol{\kappa}(w)=\left\{\begin{array}{c}
\frac{\partial^{2} w}{\partial x^{2}} \\
\frac{\partial^{2} w}{\partial y^{2}} \\
2 \frac{\partial^{2} w}{\partial x \partial y}
\end{array}\right\}, \\
\mathbf{C}=\left[\begin{array}{lll}
1 & \mu & 0 \\
\mu & 1 & 0 \\
0 & 0 & \frac{1}{2}(1-\mu)
\end{array}\right],
\end{gathered}
$$

where $w$ denotes the plate deflection of the mode shape. Equation (12) indicates that the eigenvalue sensitivity with respect to the design variable is quadratically proportional to the curvature vector. The elements with higher curvature can give rise to higher eigenvalue.

From (9), the magnitudes of modal strains are linearly correlated to the curvature vector of mode shapes in the local coordinate; that is,

$$
\phi_{\varepsilon}^{e}=-z \cdot \boldsymbol{\kappa} .
$$

Substituting (14) into (12), the eigenvalue sensitivity with respect to one of the element thicknesses, $b_{i}$, can be derived as

$$
\frac{\partial \lambda}{\partial b_{i}}=\iint_{\Omega_{i}}\left[\frac{E b_{i}^{2}}{4 z^{2}\left(1-\mu^{2}\right)} \phi_{\varepsilon}^{e T} \mathbf{C} \phi_{\varepsilon}^{e}-\lambda \rho w^{2}\right] d \Omega
$$

From (15), the coefficient of modal strain term is always positive, and hence the eigenvalue sensitivity with respect to each element thickness can be positively correlated to the modal strain term $\phi_{\varepsilon}^{e T} \mathbf{C} \phi_{\varepsilon}^{e}$ of the corresponding finite element. Therefore, thickness modification of the finite elements with higher modal strains can be more effective for design of modal frequencies of the whole structure. The relationships between the SMSs, CMSs, and eigenvalue sensitivity can be illustrated as in Figure 1.

The elements with higher modal strain at a specific mode contribute much more to the eigenvalue than elements with lower modal strain. This law provides a fast routine that one can modify the high-modal-strain elements with priority in order to obtain a desired modal frequency. It is to note that strain mode shapes can be conveniently generated and visualized by using finite element modal analysis.

\section{Numerical Investigation}

3.1. Material Distribution Problem. In the previous study, Pedersen [14] maximized the first natural frequency of a rectangular plate that is clamped at all four edges or simply supported at all four edges using topology optimization. The dimensions of the plate are $150 \mathrm{~mm} \times 100 \mathrm{~mm} \times 1.22 \mathrm{~mm}$. The material properties were Young's modulus $E=70 \mathrm{GPa}$, Poisson's ratio $\mu=0.3$, and mass density $\rho=2700 \mathrm{~kg} / \mathrm{m}^{3}$. A nonstructural mass $0.003 \mathrm{~kg}$ was placed at the centre. In the simulation of present study, the flat plate is modelled using quadrilateral shell element (S4R) in Abaqus software [35].

The material volume of topology optimization was constrained to be less than $60 \%$ in the Reference. The result of topology optimization from the clamped case is shown in Figure 2(a). In current modal analysis, Lanczos method is 


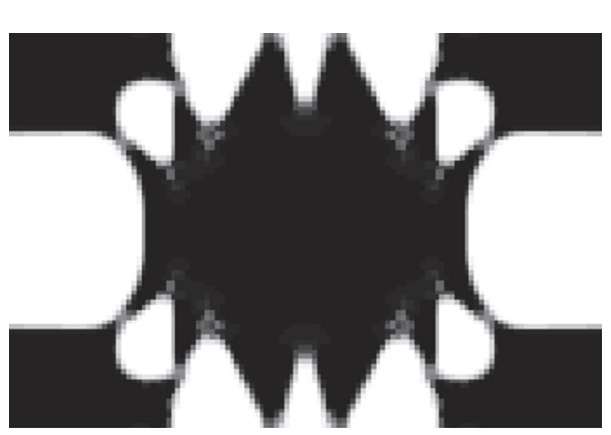

(a)

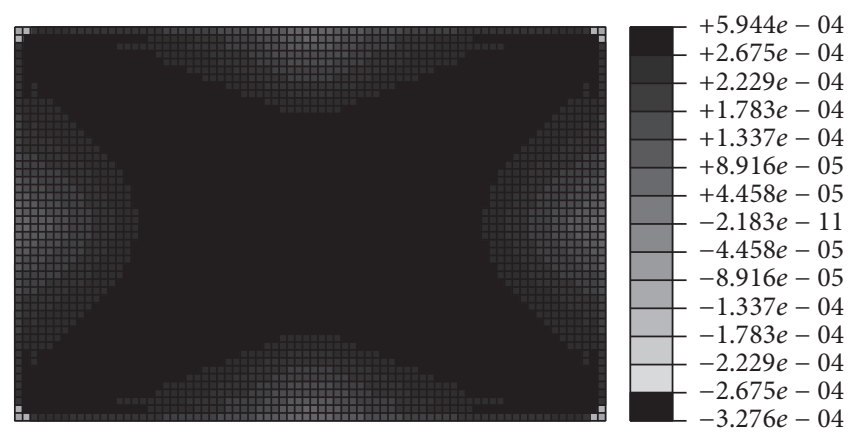

(b)

FIgURE 3: Topology optimization result and the modal principal strain of a simply supported plate: (a) topology optimization result from [14]; (b) modal principal strain.

chosen as eigensolver, and the displacement normalization is applied in eigenvector normalization (these two are used throughout the latter simulations). The black areas represent the optimal material distribution for maximizing the first eigenvalue in Figure 2(a). In Abaqus software, the modal strain distribution within the structural domain can be clearly visualized, which makes it simple and convenient to determine the regions of high modal strain in the structure for different modes. By generating the maximum in-plane principal strain (Abs) in Abaqus visualization, Figure 2(b) gives the contour of the maximum in-plane principal strain at the first mode. As the maximum magnitude is $1.249 \times 10^{-3}$, the cutoff value is set to $3.747 \times 10^{-4}$, which is $\approx 30 \%$ of the maximum magnitude. That is, the regions with the absolute magnitude of the modal strain exceeding the cutoff value are displayed in pure black. The black regions indicate the highly distributed principal strain at this mode shape, which are located at the centre of the plate and the mid of the four edges. This result is in much agreement with the topology optimization.

The corresponding results of the case with a simply supported boundary condition are shown in Figure 3. The maximum magnitude of the in-plane principal strain is 5.944 $\times 10^{-4}$, and the cutoff value is set to $2.6748 \times 10^{-4}, \approx 45 \%$ of the maximum. It can be seen that the centre and the four corners of the plate can be considered as the optimal regions, based on both the topology optimization and the modal strain analysis. The two cases shown in Figures 2 and 3 show that the modal principal strain can serve as an indicator for the optimization of the material distribution of a plate structure.

Similar to the displacement mode shapes (DMSs), the SMSs depend on the properties of mass and stiffness of a structure. Highly strained regions at a specific mode shape can make a higher contribution to the global stiffness of that mode. However, the DMSs cannot reveal this law. This is selfexplanatory for a cantilever beam: large deflection occurs at the free end with small strain but large strain at the fixed end with small deflection.

3.2. Modal Strain Characteristics of Thin Plates. In this section, some characteristics of SMSs in a flat plate, as shown in Figure 4, are discussed. The in-plane size of the flat plate is

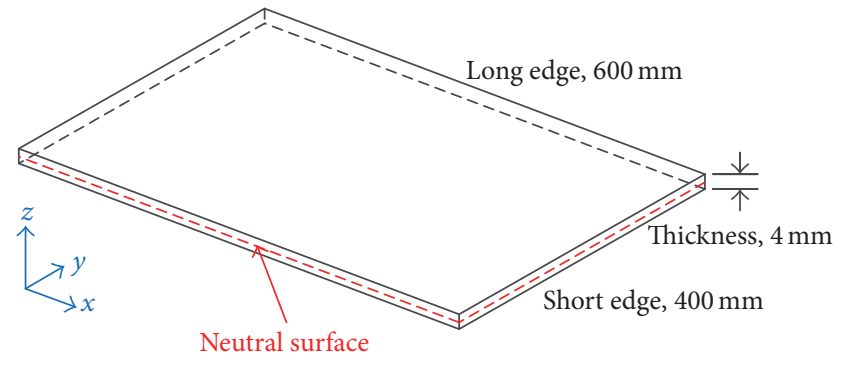

Figure 4: Model of the rectangular flat plate.

$600 \mathrm{~mm} \times 400 \mathrm{~mm}$, with the thickness of $4 \mathrm{~mm}$ and the aspect ratio of the plate is the same as the former example $(3: 2)$. Young's modulus of the material is $E=108 \mathrm{GPa}$, Poisson's ratio is $\mu=0.34$, and the mass density is $\rho=4.5 \times 10^{3} \mathrm{~kg} / \mathrm{m}^{3}$.

As the DMSs can have $x$-, $y$-, and $z$-component and magnitude shapes, the SMSs can have different contours depending on the strain components. For comparison purpose, the maximum values of the three in-plane components of the modal strains in the simply supported flat plate are depicted in Figure 5 for the first five modes. Herein, the maximum displacements are normalized to unity, and then the SMSs are generated based on the corresponding DMSs. The $x$-component of the modal strains at the 4 th order is higher than the other four orders, of which the reason is that the 4 th mode of the flat plate is the $3 \times 1$ bending mode in $x$ - $y$-plane. The $y$-components of the modal strains at the $3 \mathrm{rd}$ and 5th orders are the two highest modes, which is because the 3 rd and 5th orders are the $1 \times 2$ and $2 \times 2$ bending modes in $x-y$-plane, respectively. For the in-plane shear component, the modal strains increase as the order of mode goes up.

As mentioned in Section 2.2, the principal strain is of much importance due to its property of frame-independence. The maximum in-plane principal strains for the first five modes were extracted for the simply supported flat plate. Experimentally, the mass-normalization of the DMSs and SMSs cannot be performed with strain modal parameters only [20], unless the special strategy is used, for example, the mass-change strategy [21]. In present study, the aim of using a specific order of strain mode shape is to give a qualitative 

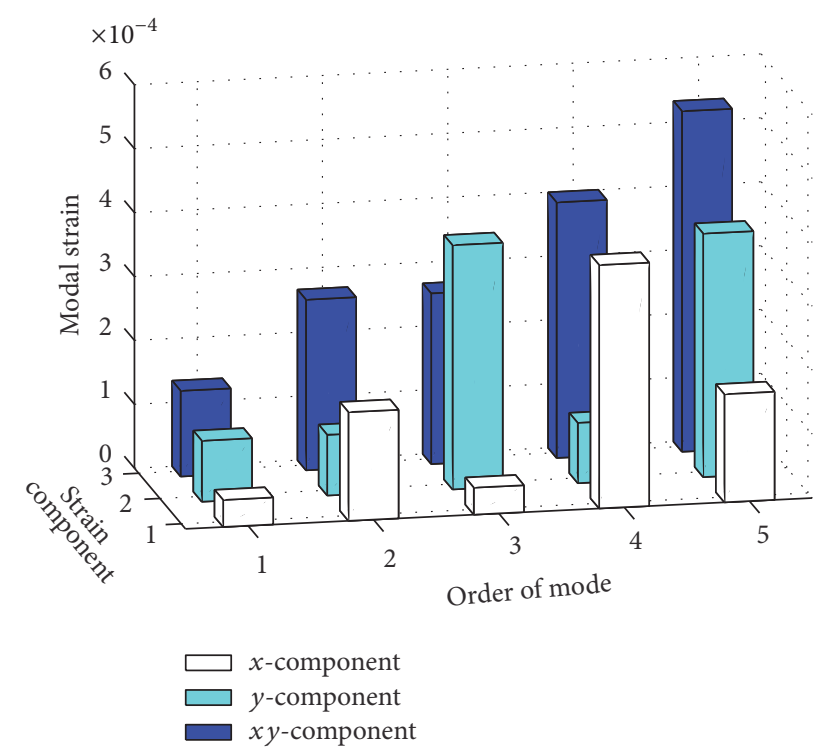

FIGURE 5: Maximum modal strains of the in-plane components of the simply supported flat plate.

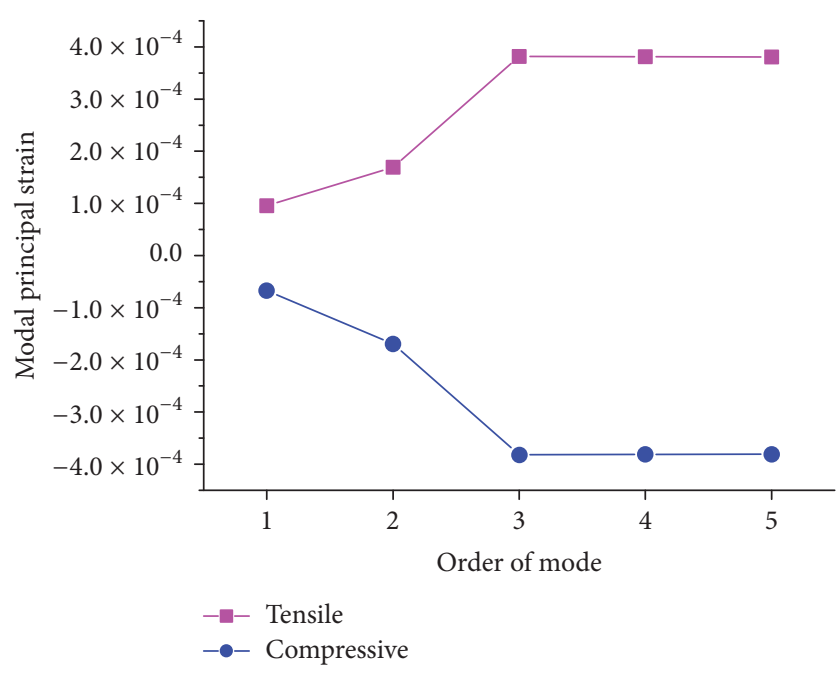

Figure 6: Tensile and compressive principal strains of the simply supported flat plate.

image of the strain intensity of the structure loaded at that modal frequency. For comparison of the relative magnitudes, it is proper to use a uniform normalization method for different modes. Therefore, in numerical modal analysis, the maximum magnitudes of the DMSs are normalized to unity, and then the SMSs are generated. The positive and negative maximum principal strains at each mode denote the tensile and compressive components of strains, respectively. The two peak values of each mode are shown in Figure 6. At the 1st order of mode, the magnitude of the positive is higher than the negative. However, due to the symmetry or antisymmetry of the 2 nd to 5 th modes, the magnitudes of the positives and the corresponding negatives are identical. Furthermore, the magnitudes of principal strains increase as the order of mode

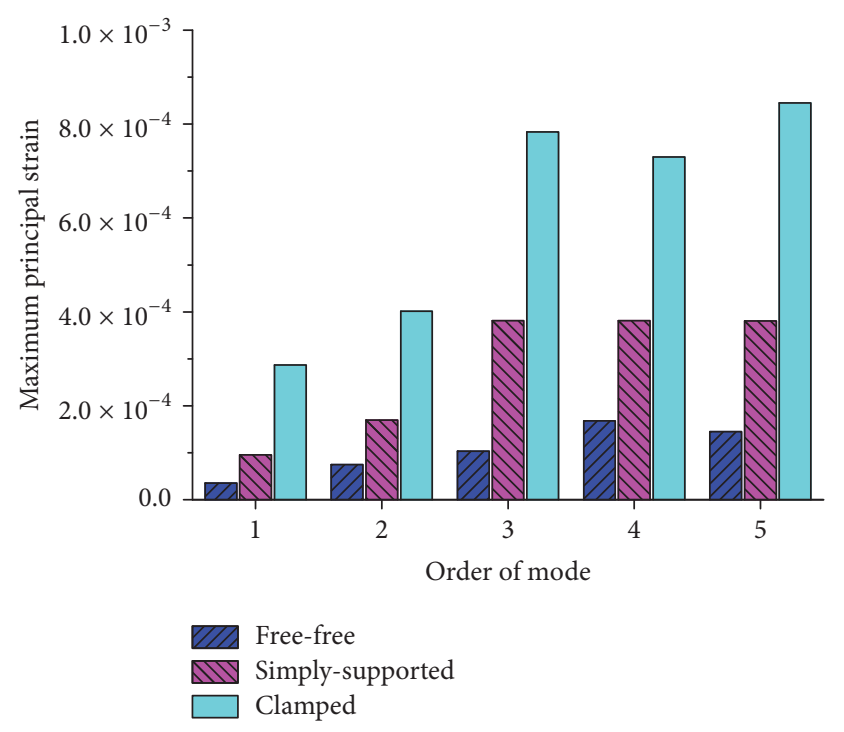

FIGURE 7: Maximum modal principal strains of the flat plates with different boundary conditions.

goes up, except that the strain magnitudes from 3 rd to 5 th orders of mode remain at the same level.

The effect of boundary conditions on the modal strain is being investigated. Three different boundary conditions were considered for the flat plate, that is, free-free supported, simply supported, and the clamped one. Figure 7 shows the positive maximum values of the in-plane modal principal strains from the 1st to 5 th modes. The maximum displacement in each mode is normalized to unity; however, the maximum modal strain of each mode varies to each other. From the first five modes, it can be seen that the modal strains increase as the constraints of the boundary conditions become tighter. From this characteristic of the SMSs, it can be found that the improvement of boundary constraints can probably increase the strain/stress concentrations in a vibrating structure, although the global stiffness can be improved via this practice. Therefore, it is worthwhile to note this characteristic when dynamic failure or vibration fatigue of a structure is of primary concern.

For validation purpose, the first strain mode shape of the flat plate is used to perform a thickness modification in order to improve the fundamental frequency of the plate. According to the first strain mode shape shown in Figure 8, the high modal principal strains are distributed at the middle of the long edges. Thus, these regions are selected to increase the thickness from $4 \mathrm{~mm}$ to $5 \mathrm{~mm}$, that is, the blue elements shown in Figure 9. The fundamental frequency of the initial plate is $161.66 \mathrm{~Hz}$. After modification, the first natural frequency is increased to $173.33 \mathrm{~Hz}$ via finite element analysis; and the result by using (15) is $171.89 \mathrm{~Hz}$. Results show the same tendency between the finite element analysis and the sensitivity equation in modal frequency modification.

3.3. Design of Stiffeners. The design of stiffeners is an effective approach to enhance the modal frequencies of plate-like structures. A stiffened rectangular plate is investigated in this 


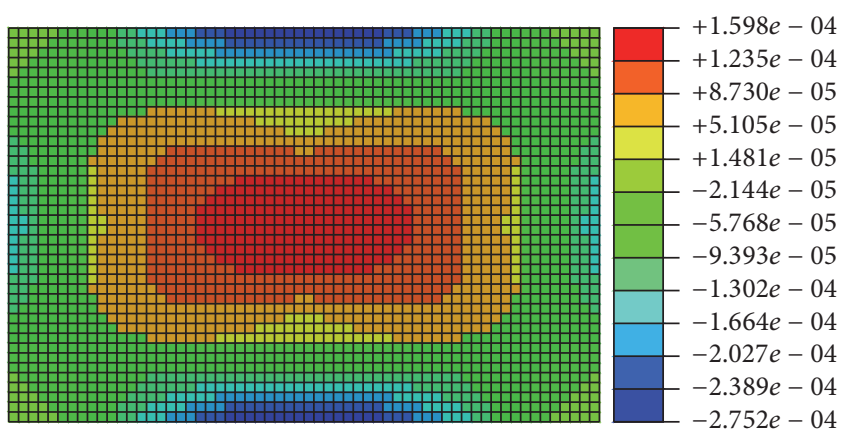

FIGURE 8: The first modal principal strain of the clamped flat plate.

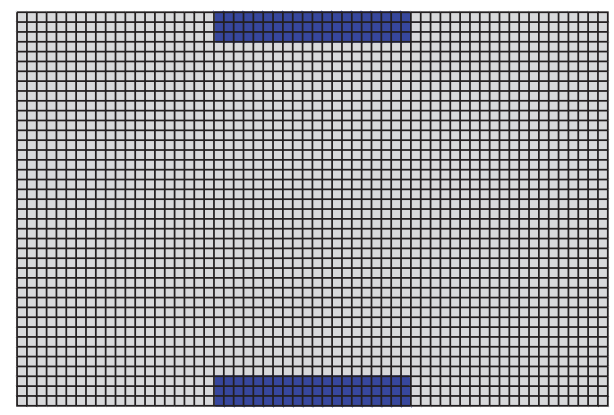

FIGURE 9: Thickness-modified model of the clamped plate.

section. It is noted that the efficiency of stiffeners depends largely on the locations and orientations of the stiffeners. In order to improve the efficiency of stiffeners, topology optimization of stiffeners of the plate was conducted [36]. The model of the stiffened plate is shown in Figure 10. The plate has the same dimensions as the flat one. The initial form of the stiffeners was based on one of the most common layouts, with three short-edge stiffeners and one long-edge stiffener. All of the stiffeners were assumed to be the identical cross section, with $4 \mathrm{~mm}$ width and $30 \mathrm{~mm}$ height. It is because hereby the attention is fixed on the layout optimization of the stiffeners, rather than on the cross sections. The boundary condition of the stiffened plate is simply supported, with no constraint on the stiffeners. The stiffeners are modelled using B31 elements.

In the previous study [36], topology optimization was conducted to maximize the fundamental frequency of the stiffened plate subject to the volume constraint, with the stiffeners taken as the design domain. The optimal material distribution is as shown in Figure 11(a) at volume fraction of $40 \%$. It can be observed that the material density of the long-edge stiffener is rather low on the whole. High density is distributed at the three short-edge stiffeners, especially at the middle one.

To investigate the modal strains of the stiffeners, modal analysis was conducted and the distribution of modal principal strain of the stiffeners at the 1st order of mode is shown in Figure 11(b). The peak of the maximum in-plane principal strain is $1.553 \times 10^{-3}$, and the cutoff value is set to $7.4 \times 10^{-4}$ ( $\approx 48 \%$ of the peak value). The elements with the maximum principal strain exceeding the threshold value are displayed as the purple colour. From the modal strain distribution, it can

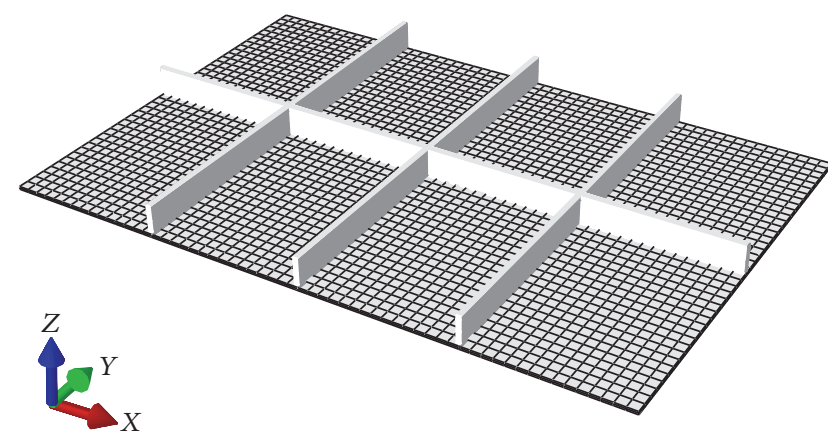

FIGURE 10: Model of the stiffened plate.

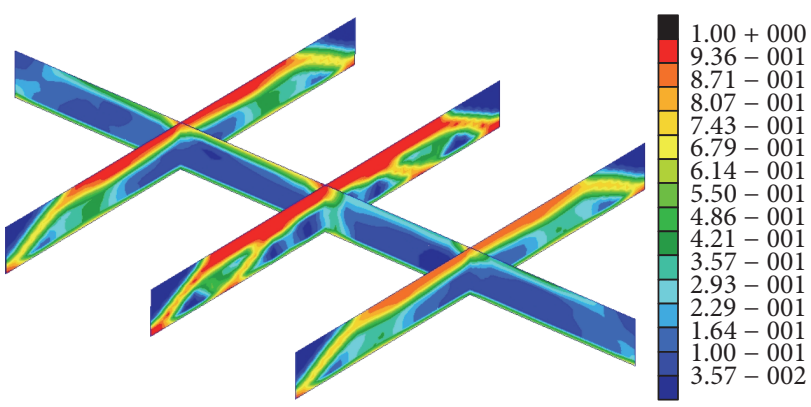

$<_{X}^{Y}$

(a)

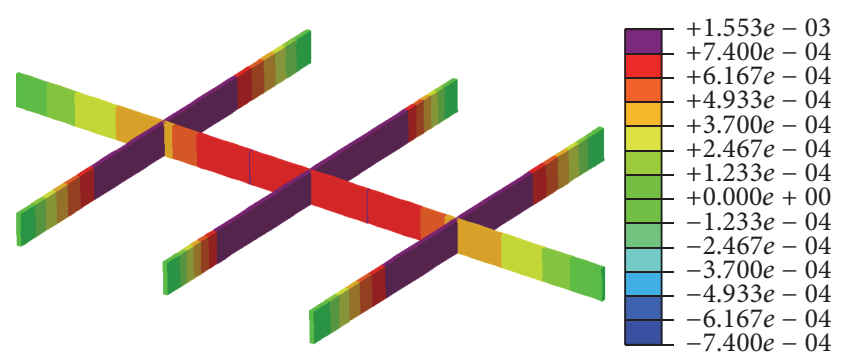

$\underbrace{Z}_{X}$

(b)

FIgURE 11: Topology optimization result and the modal strain of the stiffeners: (a) topology optimization result from [36]; (b) modal principal strain.

be seen that the area above the critical value in the long-edge stiffener is almost zero. The modal principal strains of three short-edge stiffeners are highly distributed, with the majority areas above the critical value. This result is coincident with the result of topology optimization. Therefore, it can be seen that, for a given initial layout of stiffeners, the modal strain distribution can clearly visualize the most critical regions to the associated mode. Besides, one can adjust the cutoff value to determine how large the regions will be modified in the 


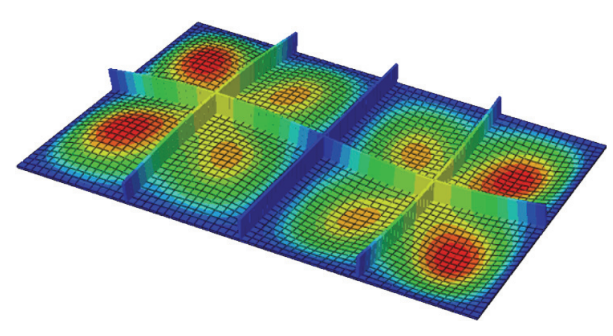

(a)

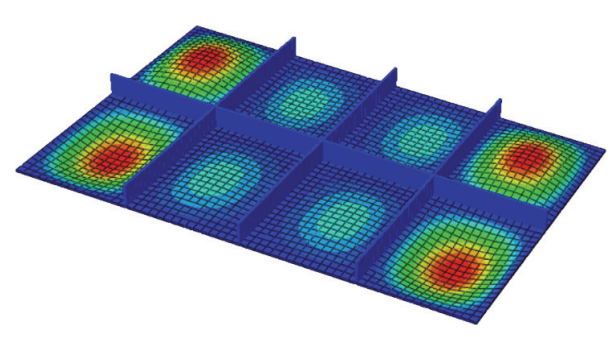

(c)

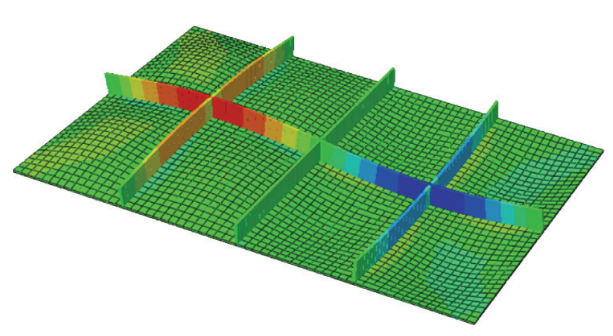

(e)

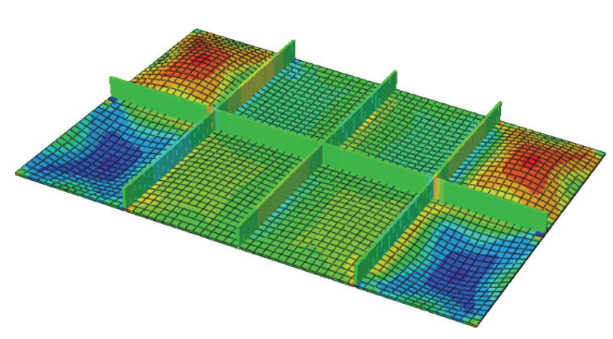

(g)

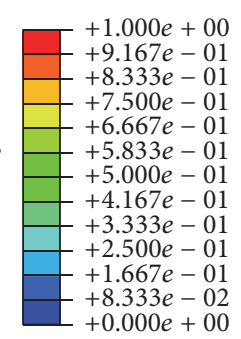

$+0.000 e+00$

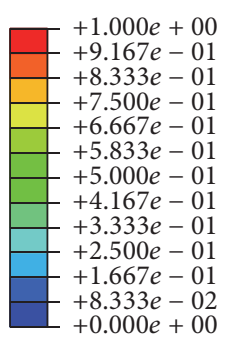

$+0.000 e+00$

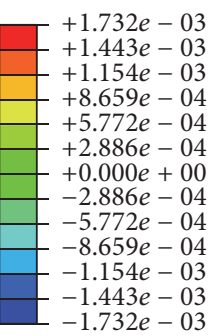

$-1.732 e-03$

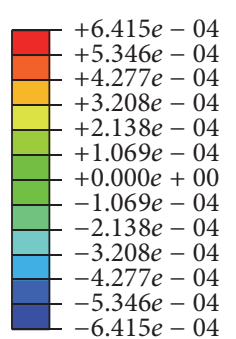

$-6.415 e-04$

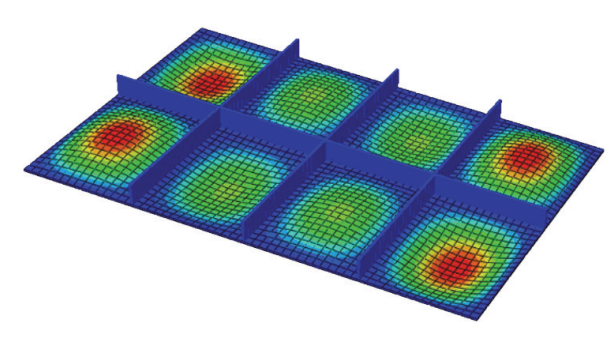

(b)

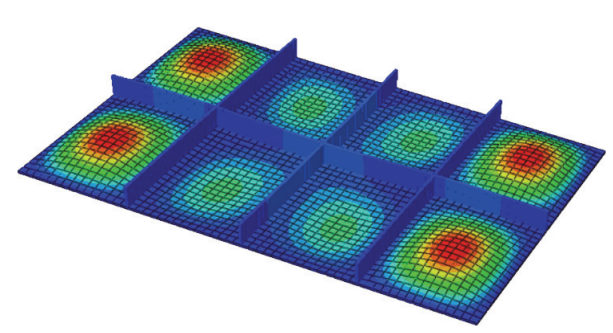

(d)
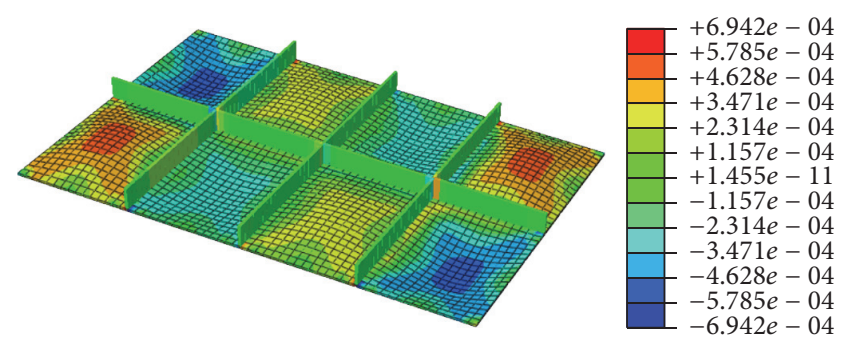

(f)
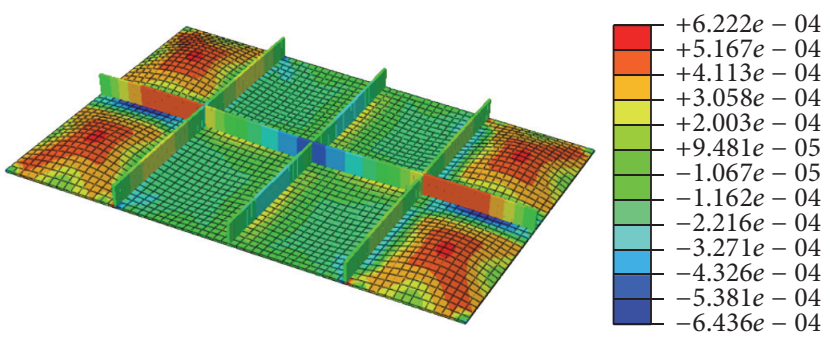

(h)

FIGURE 12: Contours of the DMSs and SMSs at deformed shapes; (a)-(d): the 2nd to 5th orders of DMSs; (e)-(h): the 2nd to 5th orders of SMSs (DMSs: displacement mode shapes; SMSs: strain mode shapes).

initial structure. Then fast modification of the highly strained regions can be performed to obtain an efficient design.

Furthermore, four higher orders of both DMSs and SMSs are compared as shown in Figure 12. The maximum magnitudes of the DMSs are normalized to unity, and then the SMSs are generated. All of the contours of both DMSs and SMSs are plotted at the deformed displacement mode shapes, with scale factor of 20 to visualize the displacement obviously. Highly strained elements can be directly observed from the SMSs of each mode. For instance, in the 2nd mode, the high modal strains are located at the $1 / 4$ and $3 / 4$ of the long-edge stiffener and the midpoint of the two side shortedge stiffeners. For the 3rd and 4th modes, all of the stiffeners are low strain distributed, due to the local modes. Thus, designers can identify the highly contributed regions to the modal stiffness from the SMSs and find ways to compress the local modes, which can often give rise to severe fatigue damage in dynamic structures.

For verification purpose, structural dynamic modification of stiffeners is conducted based on the SMSs information, considering the 2 nd modal frequency of interest. The design objective is to increase the 2 nd modal frequency by modifying the stiffener width. From Figure 12(e), it can be found that the modal strain of the 2nd mode is highly distributed in the long-edge stiffener. Therefore, the long-edge stiffener can be the most efficient region to modify for the design of the 2nd modal frequency. The width of the long-edge stiffener is modified from $4 \mathrm{~mm}$ to $8 \mathrm{~mm}$, and modal frequencies 


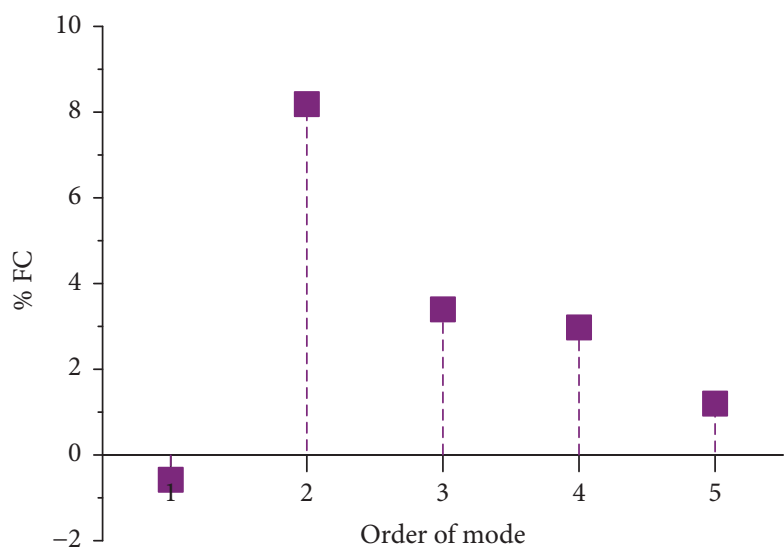

FIGURE 13: \% FC results of the first five modes.

are calculated for the modified stiffened plate. In order to evaluate the influence of widening of the long-edge stiffener, the percentage of frequency change (\% FC) is defined as

$$
\% \mathrm{FC}=\frac{f_{\text {modified }}-f_{\text {initial }}}{f_{\text {initial }}} \times 100,
$$

where $f_{\text {initial }}$ and $f_{\text {modified }}$ are the modal frequencies of the initial stiffened plate and the modified one, respectively. The \% FC results of the first five modes are shown in Figure 13. Results show that the 2nd modal frequency is most significantly improved than the other modes, which verifies that structural dynamic modification using SMSs information is effective.

\section{Conclusions}

This paper investigates the application of modal strain theory in the structural dynamic design. A modal strain based method is proposed for the design of natural frequencies of plate-like structures. The capability of determining the highly sensitive regions using modal strain analysis is theoretically demonstrated based on the eigenvalue sensitivity.

In numerical simulations, finite element models were constructed. Firstly, the application of the proposed method was conducted on a central-massed flat plate which was topologically optimized in the Reference. The results of modal principal strains show good agreement with the result from topology optimization. Furthermore, some features of the SMSs of the flat plate are investigated. Finally, the SMSs were applied to the optimization of a stiffened plate. Attention is focused on the SMSs distribution in the stiffeners, which also shows good agreement with the result from the topology optimization in the previous study. Several higher orders of SMSs were extracted, which can visualize the most sensitive regions to the corresponding modal frequency. In summary, the simulations validate the correctness and convenience of applying modal strain theory to dynamic design of plate structures. Therefore, it can be used as a quick-design tool in an industrial environment for preliminary design and optimization for dynamic consideration.

\section{Competing Interests}

The authors declare that they have no competing interests.

\section{Acknowledgments}

This work was jointly supported by the funds of the National Natural Science Foundation of China (no. 11572086 and no. 11402052); the New Century Excellent Talent in University (NCET-11-0086); the Fundamental Research Funds for the Central Universities and the Scientific Research Innovation Program of Jiangsu Province College Postgraduate (KYLX_0093); the China Scholarship Council (201506090047); and Ministry of Science and Technological Development, Serbia (TR 35011 and ON 74001).

\section{References}

[1] W. Sun, J. Zhou, D. Gong, and T. You, "Analysis of modal frequency optimization of railway vehicle car body," Advances in Mechanical Engineering, vol. 8, no. 4, 2016.

[2] Z. Fang and L. Zheng, "Topology optimization for minimizing the resonant response of plates with constrained layer damping treatment," Shock and Vibration, vol. 2015, Article ID 376854, 11 pages, 2015.

[3] D. Seung Cho, B. Hee Kim, J.-H. Kim, N. Vladimir, and T. Muk Choi, "Frequency response of rectangular plate structures in contact with fluid subjected to harmonic point excitation force," Thin-Walled Structures, vol. 95, pp. 276-286, 2015.

[4] R. Martinez-Val and E. Perez, "Aeronautics and astronautics: recent progress and future trends," Proceedings of the Institution of Mechanical Engineers, Part C: Journal of Mechanical Engineering Science, vol. 223, no. 12, pp. 2767-2820, 2009.

[5] J. Du and N. Olhoff, "Topological design of freely vibrating continuum structures for maximum values of simple and multiple eigenfrequencies and frequency gaps," Structural and Multidisciplinary Optimization, vol. 34, no. 2, pp. 91-110, 2007.

[6] B. Stanford and P. Beran, "Optimal thickness distributions of aeroelastic flapping shells," Aerospace Science and Technology, vol. 24, no. 1, pp. 116-127, 2013.

[7] U. Topal and Ü. Uzman, "Frequency optimization of laminated folded composite plates," Materials \& Design, vol. 30, no. 3, pp. 494-501, 2009.

[8] J. Luo and H. C. Gea, "Optimal Stiffener design for interior sound reduction using a topology optimization based approach," Journal of Vibration and Acoustics, vol. 125, no. 3, pp. 267-273, 2003.

[9] W. Akl, A. El-Sabbagh, and A. Baz, "Optimization of the static and dynamic characteristics of plates with isogrid stiffeners," Finite Elements in Analysis and Design, vol. 44, no. 8, pp. 513$523,2008$.

[10] B. Li, J. Hong, S. Yan, and Z. Liu, "Multidiscipline topology optimization of stiffened plate/shell structures inspired by growth mechanisms of leaf veins in nature," Mathematical Problems in Engineering, vol. 2013, Article ID 653895, 11 pages, 2013.

[11] N. Trišović, "Eigenvalue sensitivity analysis in structural dynamics," FME Transactions, vol. 35, no. 3, pp. 149-156, 2007.

[12] D. Wang, "Sensitivity analysis and shape optimization of a hole in a vibrating rectangular plate for eigenfrequency maximization," Journal of Engineering Mechanics, vol. 138, no. 6, pp. 662674, 2012. 
[13] Z.-S. Liu, J. S. Hansen, and D. C. D. Oguamanam, "Eigenvalue sensitivity analysis of stiffened plates with respect to the location of stiffeners," Structural Optimization, vol. 16, no. 2-3, pp. 155161, 1998.

[14] N. L. Pedersen, "Maximization of eigenvalues using topology optimization," Structural and Multidisciplinary Optimization, vol. 20, no. 1, pp. 2-11, 2000.

[15] X. Huang, Z. H. Zuo, and Y. M. Xie, "Evolutionary topological optimization of vibrating continuum structures for natural frequencies," Computers \& Structures, vol. 88, no. 5-6, pp. 357$364,2010$.

[16] A. K. Pandey, M. Biswas, and M. M. Samman, "Damage detection from changes in curvature mode shapes," Journal of Sound and Vibration, vol. 145, no. 2, pp. 321-332, 1991.

[17] L. Y. Yam, T. P. Leung, D. B. Li, and K. Z. Xue, "Theoretical and experimental study of modal strain analysis," Journal of Sound and Vibration, vol. 191, no. 2, pp. 251-260, 1996.

[18] C. S. Hamey, W. Lestari, P. Qiao, and G. Song, "Experimental damage identification of carbon/epoxy composite beams using curvature mode shapes," Structural Health Monitoring, vol. 3, no. 4, pp. 333-353, 2004.

[19] Z.-C. Wang, D. Geng, W.-X. Ren, and H.-T. Liu, "Strain modes based dynamic displacement estimation of beam structures with strain sensors," Smart Materials and Structures, vol. 23, no. 12, Article ID 125045, 2014.

[20] T. Kranjc, J. Slavić, and M. Boltežar, "A comparison of strain and classic experimental modal analysis," Journal of Vibration and Control, vol. 22, no. 2, pp. 371-381, 2016.

[21] T. Kranjc, J. Slavič, and M. Boltežar, “The mass normalization of the displacement and strain mode shapes in a strain experimental modal analysis using the mass-change strategy," Journal of Sound and Vibration, vol. 332, no. 26, pp. 6968-6981, 2013.

[22] W. Hong, J. Zhang, G. Wu, and Z. Wu, "Comprehensive comparison of macro-strain mode and displacement mode based on different sensing technologies," Mechanical Systems and Signal Processing, vol. 50-51, pp. 563-579, 2015.

[23] G. C. Yao, K. C. Chang, and G. C. Lee, "Damage diagnosis of steel frames using vibrational signature analysis," Journal of Engineering Mechanics, vol. 118, no. 9, pp. 1949-1961, 1992.

[24] Y. Y. Li, L. Cheng, L. H. Yam, and W. O. Wong, "Identification of damage locations for plate-like structures using damage sensitive indices: strain modal approach," Computers \& Structures, vol. 80, no. 25, pp. 1881-1894, 2002.

[25] Y. Y. Li, "Hypersensitivity of strain-based indicators for structural damage identification: a review," Mechanical Systems and Signal Processing, vol. 24, no. 3, pp. 653-664, 2010.

[26] L. He, J. Lian, and B. Ma, "Intelligent damage identification method for large structures based on strain modal parameters," Journal of Vibration and Control, vol. 20, no. 12, pp. 1783-1795, 2014.

[27] S. Li and Z. Wu, "Modal analysis on macro-strain measurements from distributed long-gage fiber optic sensors," Journal of Intelligent Material Systems and Structures, vol. 19, no. 8, pp. 937946, 2008.

[28] T. H. Ooijevaar, L. L. Warnet, R. Loendersloot, R. Akkerman, and T. Tinga, "Impact damage identification in composite skin-stiffener structures based on modal curvatures," Structural Control \& Health Monitoring, vol. 23, no. 2, pp. 198-217, 2016.

[29] F. Pelayo, A. Skafte, M. L. Aenlle, and R. Brincker, "Modal analysis based stress estimation for structural elements subjected to operational dynamic loadings," Experimental Mechanics, vol. 55, no. 9, pp. 1791-1802, 2015.
[30] Y.Zhou, Q. Fei, and S. Wu, "Utilization of modal stress approach in random-vibration fatigue evaluation," Proceedings of the Institution of Mechanical Engineers, Part G: Journal of Aerospace Engineering, 2016.

[31] K. K. Choi and N.-H. Kim, Structural Sensitivity Analysis and Optimization 1: Linear Systems, Edited by F. F. Ling, Springer Science \& Business Media, Berlin, Germany, 2006.

[32] O. C. Zienkiewicz and R. L. Taylor, The Finite Element Method for Solid and Structural Mechanics, Elsevier, Oxford, UK, 6th edition, 2005.

[33] P. Pedersen, "On optimal orientation of orthotropic materials," Structural Optimization, vol. 1, no. 2, pp. 101-106, 1989.

[34] M. P. Bendsoe, J. M. Guedes, R. B. Haber, P. Pedersen, and J. E. Taylor, "An analytical model to predict optimal material properties in the context of optimal structural design," Journal of Applied Mechanics-Transactions of the ASME, vol. 61, no. 4, pp. 930-937, 1994.

[35] Abaqus Analysis User's Manual, Version 6.10, 2011.

[36] Y. Zhou and Q. Fei, "Dynamic design of stiffeners for a typical panel using topology optimization and finite element analysis," Advances in Mechanical Engineering, vol. 7, no. 3, pp. 1-8, 2015. 


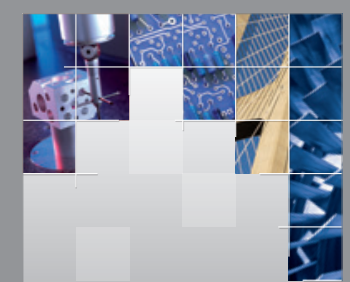

\section{Enfincering}
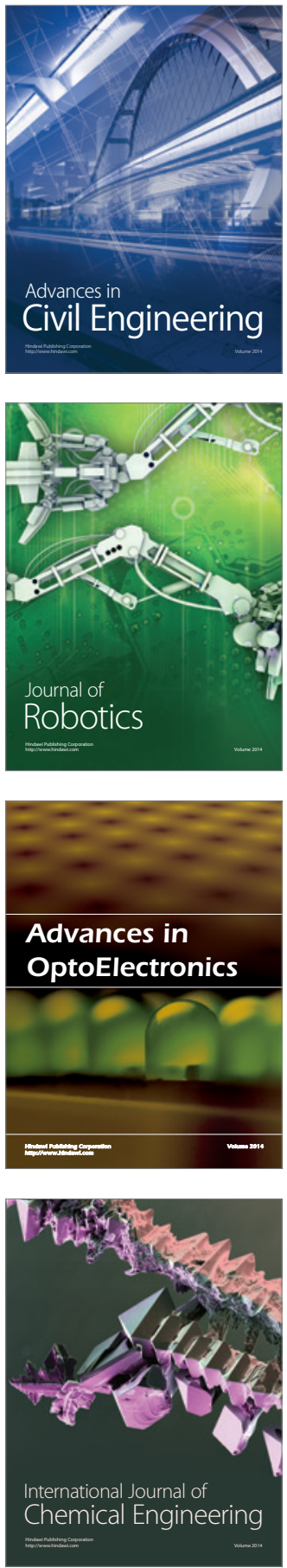

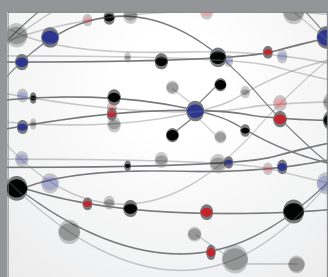

The Scientific World Journal

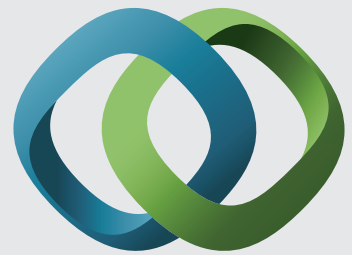

\section{Hindawi}

Submit your manuscripts at

http://www.hindawi.com
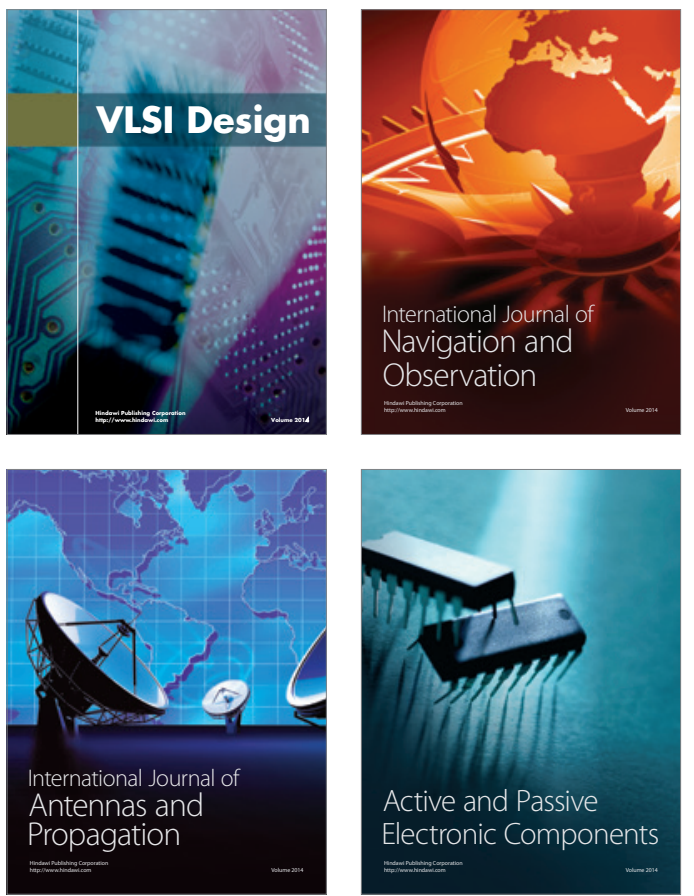
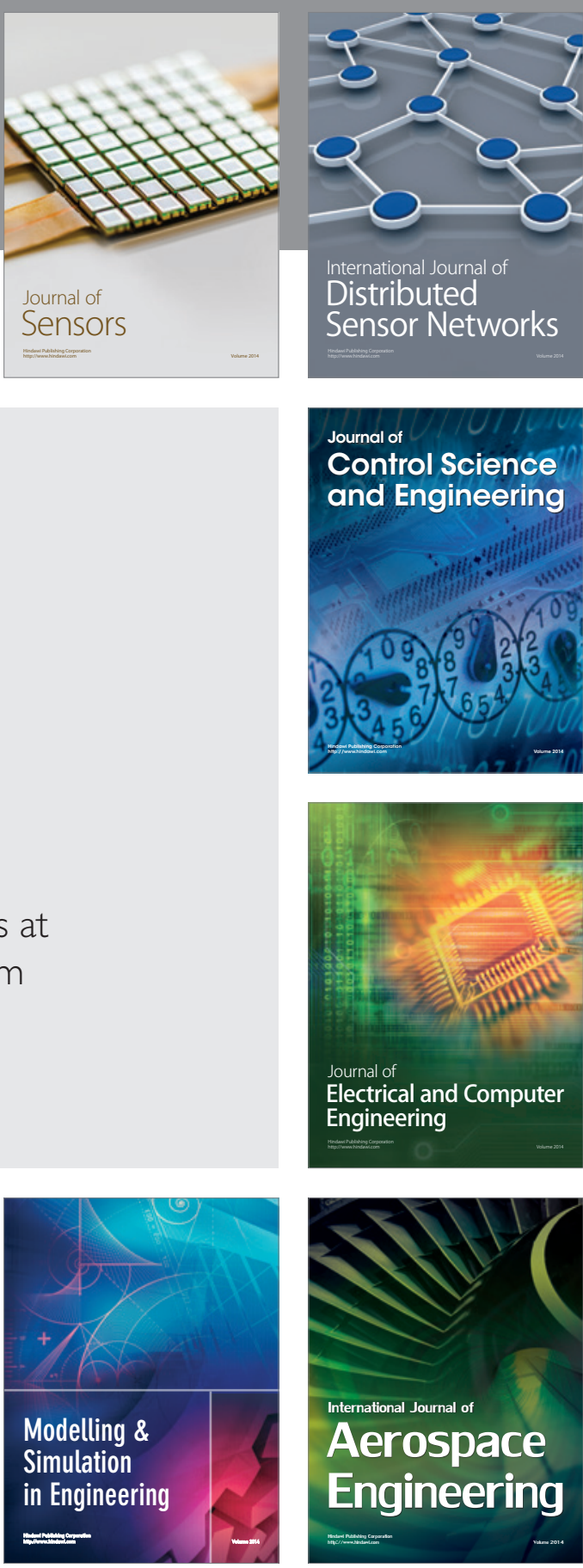

International Journal of

Distributed

Sensor Networks

Journal of

Control Science

and Engineering
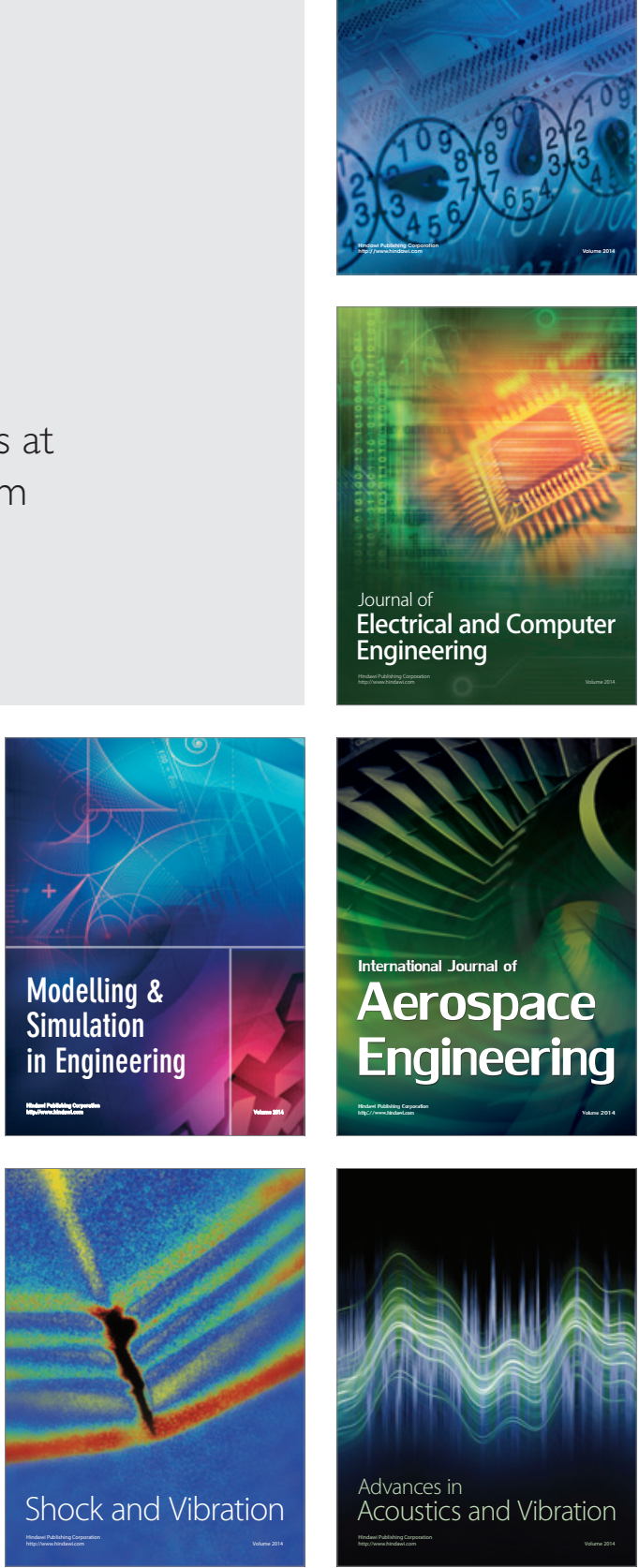